\title{
Response and Outcome of Moderate Dose Adrenocorticotropic Hormone in the Treatment of West Syndrome
}

\author{
Mahua Chandra ${ }^{1}$, Narayan Saha², Provat Kumar Sarkar ${ }^{3}$, Most. Samsun Nahar Sumi ${ }^{4}$, Shyamal Sarker ${ }^{5}$, \\ Nazmul Haque ${ }^{6}$, Banita Mistry ${ }^{7}$ \\ ${ }^{1}$ Junior Consultant, Department of Pediatric Neurology, National Institute of Neurosciences \& Hospital, Dhaka, Bangladesh; ${ }^{2}$ Professor \\ and Head, Department of Pediatric Neurology, National Institute of Neurosciences \& Hospital, Dhaka, Bangladesh; ${ }^{3}$ Assistant

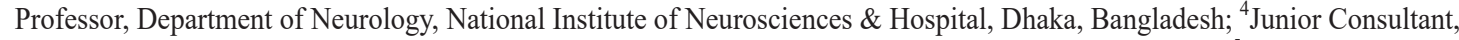 \\ Department of Pediatric Neurology, National Institute of Neurosciences \& Hospital, Dhaka, Bangladesh; ${ }^{5}$ Junior Consultant, \\ Department of Pediatric Neurology, National Institute of Neurosciences \& Hospital, Dhaka, Bangladesh; ${ }^{6}$ Assistant Professor, \\ Department of Pediatric Neurology, National Institute of Neurosciences and Hospital, Dhaka, Bangladesh; ${ }^{7}$ Assistant \\ Professor, Department of Pediatric Neurology , National Institute of Neurosciences \& Hospital, Dhaka, Bangladesh.
}

[Received on: 22 April 2021; Accepted on: 12 May 2021; Published: 1 July 2021]

\begin{abstract}
Background: West Syndrome (WS) consist of a triad of epileptic spasms, hypsarrhythmia on EEG and arrest or regression of psychomotor development. Although ACTH has been found to be effective in the treatment of WS, questions remain regarding the optimum dosage, type of ACTH, duration of therapy, and its comparative efficacy with other treatment options. Objective: To assess the response and outcome of treatment with moderate dose $\left(100 \mathrm{IU} / \mathrm{m}^{2}\right) \mathrm{ACTH}$ in children of west syndrome. Methodology: This was a prospective observational study and done over 1-year period (July 2017 to June 2018) in Pediatric Neurology OPD, National Institute of Neuroscience and Hospital (NINS\&H), Dhaka among the patients with West syndrome. All study participants were treated with moderate dose ACTH (100 unit $/ \mathrm{m} 2)(\mathrm{I} / \mathrm{M})$ and treatment completed within 12 weeks. Patients were followed up at 2, 4, 6 and 12 WKs of treatment. Results: Total 52 cases were for enrolled. In this study it was found that complete cessation of spasm $21 / 50(42 \%), \geq 50 \%$ reduction of spasm $12 / 50(24 \%),<50 \%$ reduction of spasm $8 / 50(16 \%)$ and non-responder $9 / 50(18 \%)$. At the end point of 12 weeks observation resolution of hypsarrhythmia occurred in 19/50 (38\%) cases. About 78.0\% patients developed any kind of the adverse effect. Conclusion: Moderate dose ACTH is effective in cessation of spasm and resolution of hypsarrhythmia in the studied children. [Journal of National Institute of Neurosciences Bangladesh, July 2021;7(2):108-112]
\end{abstract}

Keywords: West syndrome; response; outcome; epileptic spasm; hypsarrhythmia; ACTH

Correspondence: Dr. Mahua Chandra, Junior Consultant, Department of Pediatric Neurology, National Institute of Neuroscience and Hospital , Sher-E-Bangla Nagar, Dhaka-1207, Bangladesh; Cell no.: +8801711022930; Email: chandra.mahua@yahoo.com

Conflict of interest: There is no conflict of interest relevant to this paper to disclose.

Funding agency: This research project was not funded by any group or any institution.

Contribution to authors: Chandra M, Saha N, Sarkar PK, Sumi MSN, involved protocol preparation, data collection up to report writing. Rest are involved in designing, overall supervision and manuscript writing.

How to cite this article: Chandra M, Saha N, Sarkar PK, Sumi MSN, Sarker S, Haque N, Mistry B. Response and Outcome of Moderate Dose Adrenocorticotropic Hormone in the Treatment of West Syndrome. J Natl Inst Neurosci Bangladesh, 2021;7(2): 108-112

Copyright: (C2021. Chandra et al. Published by Journal of National Institute of Neurosciences Bangladesh. This article is published under the C-reactive Commons CC BY-NC License (https://creativecommons.org/licenses/by-nc/4.0/). This license permits use, distribution and reproduction in any medium, provided the original work is properly cited, and is not used for commercial purposes.

\section{Introduction}

West syndrome (also known as infantile spasm because of its main seizure type) is a rare form of epilepsy that begins during early infancy characterized by 3 features, namely, epileptic spasms, developmental delay, and characteristic electroencephalogram (EEG) pattern called hypsarryhythmia. West Syndrome remains to be one of the most challenging epilepsies to treat ${ }^{1}$. Prognosis of this condition depends on the etiology and adequate treatment ${ }^{2}$.

The peak age of onset of West Syndrome is 4 to 6 months, and the overall incidence of WS is 2-3 cases per 10,000 live births $^{3,4}$ with a lifetime prevalence rate of 1.5 to 2 per 10,000 children $^{5}$. There appears to be a slightly higher incidence rate in males than in females ${ }^{6}$. The optimal treatment of West Syndrome is unknown, as the 
disease is commonly refractory to many antiepileptic drugs. Adrenocorticotropic hormone (ACTH) has been reported to be an effective treatment of WS; however, it has extensive and severe adverse effect profile that deters its use. Serious adverse effects of ACTH include hypertension, infection, electrolyte abnormalities, Cushing syndrome, cardiac hypertrophy, and reversible cerebral atrophy?

The exact pathophysiology of IS remains to be elucidated, and various mechanisms have been hypothesized. The rationale behind the usage and efficacy of ACTH and oral steroids in IS is the hypothesized dysregulation of hypothalamo-pituitaryadrenal (HPA) axis inthe pathophysiology of IS, which has been tested recently ${ }^{8}$. In addition to ACTH, several other treatment options have been investigated for use in WS, including oral corticosteroids, vigabatrin, valproic acid, pyridoxine, zonisamide, intravenous immunoglobulin, topiramate, thyrotropin-releasing hormone, levetiracetam, and the ketogenic diet. With the exception of vigabatrin, these medications lack sufficient evidence to support their use for treatment of infantile spasms ${ }^{9}$. Although ACTH has been found to be effective in the treatment of WS, questions remain regarding the optimum dosage (low-dose versus high-dose), type of ACTH (synthetic vs natural), duration of therapy, and its comparative efficacy with other treatment options? American academy of pediatrics describes that typically, treatment consists of either medium (100 IU/m2 per day) or high-dose (150 IU/m2 per day) ACTH injected intramuscularly for as few as 2 weeks or as many as 2 months ${ }^{10}$.

There is a paucity of prospective studies and even fewer randomized or controlled treatment trials in this disorder ${ }^{11}$. Therefore, this present study was undertaken to see the response and outcome of once daily moderate dose ACTH in the treatment of West Syndrome.

\section{Methodology}

This hospital based prospective observational study was done in the Outpatient and inpatient department of Pediatric Neurology at National Institute of Neuroscience and Hospital, Sher-E-Bangla Nagar, Dhaka, Bangladesh from July 2017 to June 2018. Patient of West syndrome, age of more than 2 month to 2 years, diagnosed by history presence of clinical spasm that occur mainly in clusters either flexor, extensor or both or asymmetric by direct observation or video recording of the spasm along with $\mathrm{h} / 0$ developmental delay or regression, and EEG demonstrating hypsarrhythmia or modified hypsarrhythmia without prior treatment of $\mathrm{ACTH}$, prednisolone or vigabatrin therapy were included in this study. Diagnosed case of tuberous sclerosis, having infantile spasm in addition to other seizure type and having neurometabolic diseases diagnosed clinically were excluded from the study. Children were evaluated thereafter through detail history and clinical examination. History related to seizure type, frequency, age at onset, antenatal, natal and postnatal details, family history, developmental history and the ongoing treatment were noted. Through general examination, systemic examination including neurological examination were done. A base line complete blood count, electrolyte, SGPT, and creatinine were obtained. Baseline CT-scan of brain and EEG were done. Informed written consent both in Bangla and English were obtained from parents or attendants after full explanation of the details of the research process. Findings of observation were recorded in a prescribed data collection form. All study participants were treated with moderate dose (100 IU/ m2) ACTH (I/M). In this study natural ACTH was used, named Acton prolongatum injection which contains carboxymethylcellulose $(1 \mathrm{~mL}=60$ Unit $)$ made by Ferring company. Dose of ACTH was used in $100 \mathrm{IU}$ per $\mathrm{m}^{2}$ per day, once daily single dose for 4 weeks then 80 unit per $\mathrm{m}^{2}$ per day, every alternate day single dose for 4 weeks then 60 unit per $\mathrm{m}^{2}$ per day, twice weekly single dose for next 4 weeks. The observational period for each patient was 12 weeks after starting of ACTH treatment. Every patient was followed at 2, 4, 6 and 12 wks. Some investigations (CBC, RBS, Serum electrolyte) were done at $2^{\text {nd }}$ and $4^{\text {th }}$ weeks. During 12 weeks of observation parents were asked to maintain a daily seizure dairy, recording frequency of seizure. Time to reduction or complete cessation of spasms after initiation of treatment, were recorded. A repeat EEG was done at 14 to 21 days or later but within 42 to 49 days after starting treatment and electrographic changes were recorded. Patient recorded daily BP chart from local pediatricians if available but otherwise BP was maintained at least weekly. All patient's blood pressure was cheeked during routine follow up of the study. At each follow up adverse effects were documented in data collection sheet. Information were recorded over phone from those case who were lost to follow up. The child who developed infection during ACTH treatment were treated properly. Data were collected, compiled and tabulated according to key variables. The analysis of different variables were done according to standard statistical analysis. Qualitative data were expressed as frequency and percentage and quantitative data were 
expressed as mean and standard deviation. Data were processed and analyzed using software Statistical Package for Social Science (SPSS) version 20.0. 95.0\% confidence interval were calculated. Mann-Whitney U test, Chi-square test and Wilcoxon signed ranks test and unpaired $t$ test were done to see the level of significance. A value of $\mathrm{p}$ less than 0.05 was considered statistically significant for all test.

\section{Results}

In this study according to the selection criteria total 52 were enrolled. Data were documented in data collection form. One patient did not complete treatment and one patient expired before completion of treatment. So, finally total 50 patients were analyzed. In this study demographic variable such as age, sex, weight, clinical variables like age of onset of spasm, type of spasm, frequency of spasm, family history of consanguinity, presence of perinatal asphyxia, developmental history before spasm, presence of microcephaly, brain abnormalities and main outcome variables like reduction of spasms, complete cessation of spasms, resolution of hypsarrhythmia and adverse events were evaluated. Mean age of the patients was $12.61 \pm 7.11$ months. Males $(58.0 \%)$ were predominant than females $(42.0 \%)$ (Table 1).

Table 1: Demographic profile of the patients $(\mathrm{n}=50)$

\begin{tabular}{lcc}
\hline Variables & Frequency & Percent \\
\hline Age Group & & \\
- $\quad \leq 12$ months & 29 & 58.0 \\
- $\quad 13$ to 24 months & 21 & 42.0 \\
Mean \pm SD & $12.61 \pm 7.11$ & \\
Gender & & \\
- Male & 29 & 58.0 \\
\hline
\end{tabular}

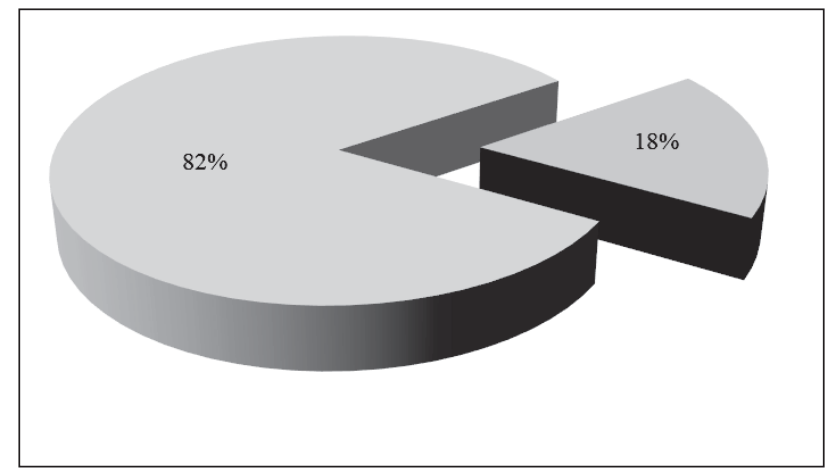

Figure I: Classification of study patients of West syndrome $(\mathrm{n}-50)$

Majority (82\%) were symptomatic and $18 \%$ were either cryptogenic or idiopathic (Figure I).
Table 2: Response of treatment of study patients ( $n-50)$

\begin{tabular}{lcc}
\hline Response & Frequency & Percent \\
\hline Complete cessation of spasm & 21 & 42.0 \\
$>50 \%$ reduction & 12 & 24.0 \\
$\leq 50$ reduced & 8 & 16.0 \\
Non responder & 9 & 18.0 \\
Total & $\mathbf{5 0}$ & $\mathbf{1 0 0 . 0}$ \\
\hline
\end{tabular}

Complete cessation of spasm was achieved in $42.0 \%$, $>50 \%$ reduced in $24.0 \%$ cases, $\leq 50 \%$ reduced in $16.0 \%$ cases \& non responder was $18.0 \%$ patients (Table 2).

Table 3: Response of Treatment between symptomatic and cryptogenic West syndrome

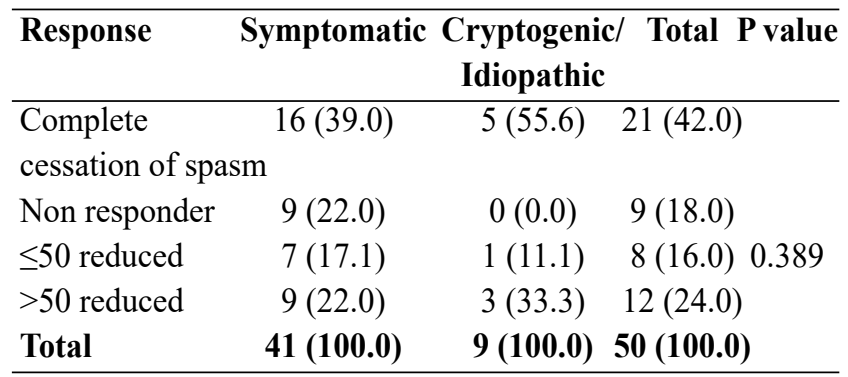

Chi-square test was done to measure the level of significance

Complete cessation of spasm occurs 16/41(39\%) in symptomatic cases and 5/9 (55\%) in cryptogenic cases there is no statistical significance $(\mathrm{p}=0.389)$. spasm $>50$ reduced $9 / 41(22 \%)$ in symptomatic cases and $3 / 9$ $(33 \%)$ in cryptogenic cases (Table 3$)$.

Table 4: Resolution of Hypsarrhythmia in EEG $(n=50)$

\begin{tabular}{lcc}
\hline Resolution & Frequency & Percent \\
\hline Absent & 19 & 38.0 \\
present & 31 & 62.0 \\
\hline
\end{tabular}

Complete resolution of hypsarrhythmia occurred in EEG 38.0\% cases after treatment (Table 4).

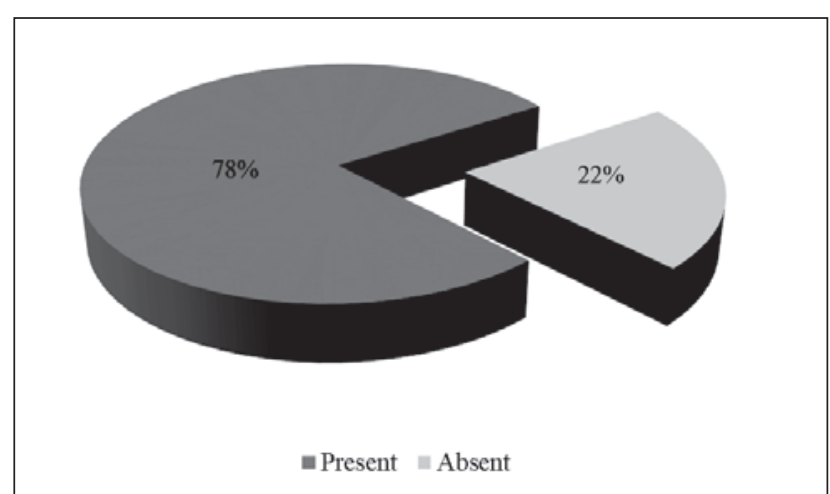

Figure II: Adverse effect during treatment

Adverse effect was found in $78.0 \%$ cases during treatment (Figure II). 
of CPSP (OR 3.4; 95\% CI 1.5-8.4; $\mathrm{p}=0.003$ ) in comparison to those with lesions in the left. Similarly, participants with ischemic stroke had higher risk of CPSP (OR 3.5; 95\% CI 1.4-9.0; $\mathrm{p}=0.007$ ) in comparison to those with hemorrhagic stroke (Table 4).

Table 5: Pattern of Adverse effect after treatment $(n=50)$

\begin{tabular}{lcc}
\hline Adverse Effect & Frequency & Percent \\
\hline Infection & 20 & 40.0 \\
Hypertension & 12 & 24.0 \\
Irritability & 35 & 70.0 \\
Sleep disturbance & 31 & 62.0 \\
Electrolyte imbalance & 3 & 6.0 \\
Hospital admission due to & 1 & 2.0 \\
adverse effect & & \\
\hline
\end{tabular}

Maximum patients had irritability (70.0\%) followed by sleep disturbance $(62.0 \%)$, infection $(40.0 \%)$, hypertension $(24.0 \%)$ and electrolyte imbalance $(6.0 \%)$ (Table 5).

\section{Discussion}

West Syndrome remains to be one of the most challenging epilepsies to treat. ${ }^{1}$ Adrenocorticotropic hormone (ACTH) has been reported to be an effective treatment of WS; however, it has extensive and severe adverse effect. In this study demographic data such as age and sex, weight were evaluated. It was found mean age of patients was $12.87 \pm 7.15$ months when they stared ACTH treatment. One study ${ }^{12}$ showed mean age of presentation was 9.4 months that was lower age than this study patients and another study ${ }^{13}$ has showed that 13.1 months that finding was nearly consistent with this study.

It was found that among study patients male were $29 / 50(55.8 \%)$ and female were $21 / 50(44.2 \%)$. Other two studies also found male preponderance $72.0 \%$ and $81.0 \%$ respectively that were also similar to our study $^{12-13}$. The reason of male preponderance may be due to more attention of family members to treat male babies.

Main outcome variables like reduction of spasms, complete cessation of spasms, resolution of hypsarrythmia and adverse events were evaluated in our study. In this study reduction of spasm were divided the by four categories such as complete cessation of spasm, $\geq 50 \%$ reduction of spasm, $\leq 50 \%$ reduction of spasm and no reduction of spasm and categorize the response by four types such as responder (complete cessation of spasm), partial response( $>50 \%$ reduction), poor response $(<50 \%$ reduction) and non-responder (no reduction of spasm).

At the end of the 12 weeks of follow up it was found that complete cessation of spasm (responder) $21 / 50(42 \%), \quad \geq 50 \%$ reduction of spasm (partial response) $12 / 50(24 \%)<50 \%$ reduction of spasm(poor response) $8 / 50(16 \%)$ and no reduction non responder $9 / 50(18 \%)$ cases. One study reviewed to determine the efficacy of various ACTH dosages of treatment response where majority of the patients got intermediate dose (60 $-100 \mathrm{IU} / \mathrm{m} \mathrm{2}$ ) and showed (17/26) 65\% cases had complete cessation of spasm. This study had higher response rate than our study ${ }^{14}$.

Complete cessation of spasm occurred $39 \%$ in symptomatic group and 55\% in cryptogenic group ( $\mathrm{p}>$ .05 ) in this study. Response was better in cryptogenic group which has a clinical significance but it was not statistically significant. If study sample size would be high then results may be statistically significant. This was also found consistent with a Japanese article ${ }^{15}$. It is difficult to compare the results of this study to this Japanese article because they use low dose synthetic subunits of ACTH which may not be biologically equivalent to corticotrophin (natural ACTH). Unfortunately our study was compared to synthetic ACTH because most of the countries use synthetic ACTH of the treatment of infantile spasm ${ }^{16-17}$.

Very few data were available using natural ACTH where they used high and low dose and low to moderate dose $\mathrm{ACTH}^{18-20}$. In addition to there is no consensus on the use of synthetic versus natural ACTH. The dosing conversion between the two products is unclear and there are no comparative studies available. For clinical purpose, $1 \mathrm{mg}$ of synthetic $\mathrm{ACTH}$ is equivalent to 100 IU of natural $\mathrm{ACTH}^{15}$.

In this study it was observed that hypsarrhythmia resolution occurred in 19/50(38.0\%) cases after treatment. Ibrahim et $\mathrm{al}^{14}$ reviewed twenty six patients and majority of the patients got intermediate dose (60 to $100 \mathrm{IU} / \mathrm{m} 2$ ) and showed resolution of hypsarrhythmia occurs in (17/26) 65.0\% cases. This study had higher hypsarrhythmia resolution rate than this present study. In this study it was found at the end point of 12 weeks observation $78.0 \%$ patients shows any kind of adverse effect and $22.0 \%$ had no adverse effect. Adverse effects are hypertension $(24.0 \%)$, infections $(40.0 \%)$ like (fever, respiratory tract infection, loose motion, soft tissue infection, oral thrush), irritability (62.0\%) and sleep disturbance $(70.0 \%)$, electrolyte imbalance $(6.0 \%)$ were present.

During treatment adverse effects were managed 
properly. Irritability and sleep disturbance were managed by proper counseling but no benzodiazepines were used because it may change the treatment response. There was no life threatening infections. Infections that developed treated with oral antibiotics. Hypertension was managed by giving tab Nifidipine. Hypertension subsided gradually during tapering of treatment. No symptomatic hypertension developed. In a randomized control trial ${ }^{13}$ comparing the effectiveness of high and low dose natural ACTH therapy the adverse effect profiles were very similar between two groups included irritability, oral thrush, infection and hypokalemia but higher incidence of hypertension $(31.0 \%$ vs $4.0 \%$ respectively).

In this study $28.0 \%$ cases develop relapse within 12 weeks of follow up after complete cessation of spasm. Ibrahim et $\mathrm{al}^{14}$ found $12 \%$ cases of relapse that was not consistent with this study. In a randomized control trial ${ }^{21}$ comparing the effectiveness of high and low dose natural ACTH therapy no significant difference relapse rates $(15.0 \%$ vs $21.0 \%$ respectively) after end of 1 year follow up; however, this present study showed high relapse rate than this study.

\section{Conclusion}

Results of the study documented that moderate dose ACTH is found to be effective in cessation of spasm and resolution of hypsarrhythmia in the studied children of west syndrome. The adverse effects are observed in majority of patients and some cases develop relapse of spasm during study period.

\section{References}

1. Song JM, Hahn J, Kim SH, Chang MJ. Efficacy of Treatments for Infantile spasm: A Systematic Review. Clinical Neuropharmacology 2017;40(2):63-84

2. Belousova ED, Shulyakova IV, Okhapkina TG. Hormonal treatment in west syndrome. Zh Nevrol Psikhiatr Im S S Korsakova 2016;116(9):61-66

3. Riikonen R. Epidemiological data of West syndrome in Finland. Brain Dev 2001;23:539-41

4. Pavone P, Striano P, Falsaperla R, Pavone L, Ruggieri M. Infantile spasms syndrome, West syndrome and related phenotypes: what we know in 2013. Brain and development. 2014;36(9):739-51
5. Jia JL, Chen S, Sivarajah V, Stephens D, Cortez MA. Latitudinal differences on the global epidemiology of infantile spasms: systematic review and meta-analysis. Orphanet Journal of rare diseases. 2018;13(1):1-7

6. Tsao CY. Current trends in the treatment of infantile spasms. Neuropsychiatric disease and treatment. 2009;5:289

7. Stafstrom CE, Arnason BG, Baram TZ, Catania A, Cortez MA, Glauser TA, Pranzatelli MR, Riikonen R, Rogawski MA, Shinnar S, Swann JW. Treatment of infantile spasms: emerging insights from clinical and basic science perspectives. Journal of child neurology. 2011;26(11):1411-21

8. Yang G, Zou LP, Wang J, Ding YX. Epigenetic regulation of glucocorticoid receptor and infantile spasms. Med Hypotheses 2011;76:187-9

9. Go CY, Mackay MT, Weiss SK, Stephens D, Adams-Webber T, Ashwal S., et al. Evidence-based guideline update: Medical treatment of infantile spasms Neurology. 2012; 78(24): 1974-1980 10. Kossoff EH. Infantile spasms. The neurologist. 2010;16(2):69-75

11. Hancock EC, Osborne JP, Edwards SW. Treatment of infantile spasms. 2013;5(6):CDOO1770

12. Khreisat HW. Clinical Profile of Infants with Hypsarrhythmia. Acta Inform Med. 2011;19(3):149-52

13. Kaushik JS, Patra B, Sharma S, Yadav D, Aneja S. Clinical spectrum and treatment outcome of West Syndrome in children from Northern India. Seizure 2013; $22: 617-621$

14. Ibrahim S, Gulab S, Ishaque S, Saleem T. Clinical profile and treatment of infantile spasms using vigabatrin and ACTH-a developing country perspective. BMC pediatrics. 2010;10(1):1-9 15. Product information. Cortrosyn (cosyntrophin) injection, power for solution. Rancho Cucamonaga, CA; Amphastar Pharmaceuticals, Inc, September 2005

16. Kodama K, Omata T, Arai H, Tanabe Y.Study of the efficacy of low-dose synthetic ACTH therapy without tapering to treat West syndrome. No To Hattatsu. 2016;48(3):195-8

17. Shumiloff NA, Lam WM, Manasco KB. Adrenocorticotropic hormone for the treatment of West Syndrome in children. Annals of Pharmacotherapy. 2013;47(5):744-54

18. Hodgeman RM, Kapur K, Paris A, Marti C, Can A, Kimia A, Loddenkemper A, et al Effectiveness of once-daily high-dose ACTH for infantile spasms. Epilepsy \& Behavior $2016 ; 59: 4-8$

19. Riikonen R. The latest on infantile spasms. Current opinion in neurology. 2005;18(2):91-5.

20. Wanigasinghe J, Arambepola C, Sri Ranganathan S, Sumanasena S, Muhandiram EC. The efficacy of moderate-to-high dose oral prednisolone versus low-to-moderate dose intramuscular corticotropin for improvement of hypsarrhythmia in West syndrome: a randomized, single-blind, parallel clinical trial. Pediatr Neurol. 2014;51(1):24-30

21. Kossoff EH, Hedderick EF, Turner Z, Freeman JM. A case-control evaluation of the ketogenic diet versus ACTH for new-onset infantile spasms. Epilepsia. 2008;49(9):1504-9 